\title{
Nuclear medium modifications of properties of kaons measured around threshold with FOPI
}

\author{
K. Piasecki (FOPI Collaboration) ${ }^{* \dagger}$ \\ University of Warsaw, Faculty of Physics \\ E-mail: krzysztof.piasecki@fuw.edu.pl
}

\begin{abstract}
We report on the investigation of modifications of basic properties of $\mathrm{K}^{+}$and $\mathrm{K}^{-}$mesons emitted from collisions of $\mathrm{Ni}+\mathrm{Ni}$ at beam energy of $1.91 \mathrm{~A} \mathrm{GeV}$. Experimental $\mathrm{K}^{-} / \mathrm{K}^{+}$ratio are presented in a wide range of phase space parameterized by kinetic energy and emission angle in the nucleon-nucleon centre of mass. The $v_{1}$ component of the azimuthal distribution was extracted as a function of rapidity and transverse momentum for central, and semi-peripheral collisions. A comparison of these patterns with the HSD transport model favours the existence of the kaonnucleon in-medium potential. For the IQMD model, this interaction scenario is confirmed in case of $\mathrm{K}^{-}$, whereas for $\mathrm{K}^{+}$the picture is less clear.
\end{abstract}

55th International Winter Meeting on Nuclear Physics

23-27 January, 2017

Bormio, Italy

* Speaker. 


\section{Introduction}

Effects of modifications of basic properties of hadrons (like mass and decay constant) propagating through the hot and/or dense nuclear medium are the subject of intensive studies throughout last 30 years $[1,2,3,5]$. In particular, various theoretical approaches predict a somewhat repulsive interaction of $\mathrm{K}^{+}$and $\mathrm{K}_{s}^{0}$ meson with nucleon, whereas the interaction of antikaon with nucleon is predicted to be strongly attractive. Historically, the kaon-nucleon interaction was first represented in terms of an additional potential $U_{\mathrm{KN}}$ (positive/negative for repulsion/attraction, respectively) [3]. However, more recent approaches based on the chiral effective field theory with coupled channels predict that the in-medium spectral function of antikaon gains significant width and becomes momentum-dependent [5]. While these modifications cannot be measured directly, they are predicted to alter observables like kinetic energy spectrum or flow (anisotropy of azimuthal distribution with respect to the reaction plane). During the collision of heavy ions the baryonic matter is found to exhibit strong flow effects. If a kaon (antikaon) propagates through such a medium, it is expected to be repelled from (resp. attracted to) the centers of density, and therefore, its flow pattern deviates (resp. gets similar to) that of the bulk of baryonic matter. On the other hand, as kaons leave the collision zone, their spectral functions have to return to the vacuum form. If the antikaon leaves the collision zone, and its effective mass is to increase, the simplest way to acquire extra energy is to decelerate. For the kaon, the effect should be the opposite.

In spite of the importance of the strangeness production to the understanding of the nucleusnucleus collisions around threshold energies, the experimental knowledge on the in-medium effects of kaons has been quite limited. The ratios of $\mathrm{K}^{-} / \mathrm{K}^{+}$as function of kinetic energy, compared to the predictions of the (R)BUU transport models [6, 7] or HSD models [8] were strongly suggesting the non-zero $U_{\mathrm{KN}}$ potentials, however, these ratios were obtained for narrow windows of the polar emission angle [6,7], and momentum [8]. The comparisons of experimentally obtained flow patterns of $\mathrm{K}^{+}$emission to the predictions of the (R)BUU transport model $[9,10]$ were also displaying preference for the in-medium effects, however, again, the data were investigated experimentally in narrow windows of phase space, and the data volume allowed to obtain results with significant uncertainties. In addition, throughout the last decade the yield of the important feeding of the $\phi$ meson decays into the emitted negative kaons was experimentally constrained to be $18 \pm 3 \%$ [11, 12]. While the energy spectrum of negative kaons originating from decays of $\phi$ mesons seems to be considerably softer than that of $\mathrm{K}^{-}$emitted directly from the collision zone $[13,8]$, the production and decays of $\phi$ mesons within the abovementioned transport models was either not implemented yet, or did not match the experimental findings.

In addition, a very recent prediction of the UrQMD transport model calculations on the kinetic energy profile of $\mathrm{K}^{-} / \mathrm{K}^{+}$ratio from $\mathrm{Al}+\mathrm{Al}$ collisions at $1.9 \mathrm{~A} \mathrm{GeV}$ was found to nicely agree with the experimental profile [14], despite the fact that no in-medium modifications of kaon properties are implemented within this model. However, the $\mathrm{K}^{-}$and $\mathrm{K}^{+}$energy spectra compared separately exhibit some excess with respect to the experiment.

Therefore more precise data, covering wider range of phase space appear to be needed to clarify the situation. Data presented in this proceedings paper aims to bring the problem a step closer to the solution. 


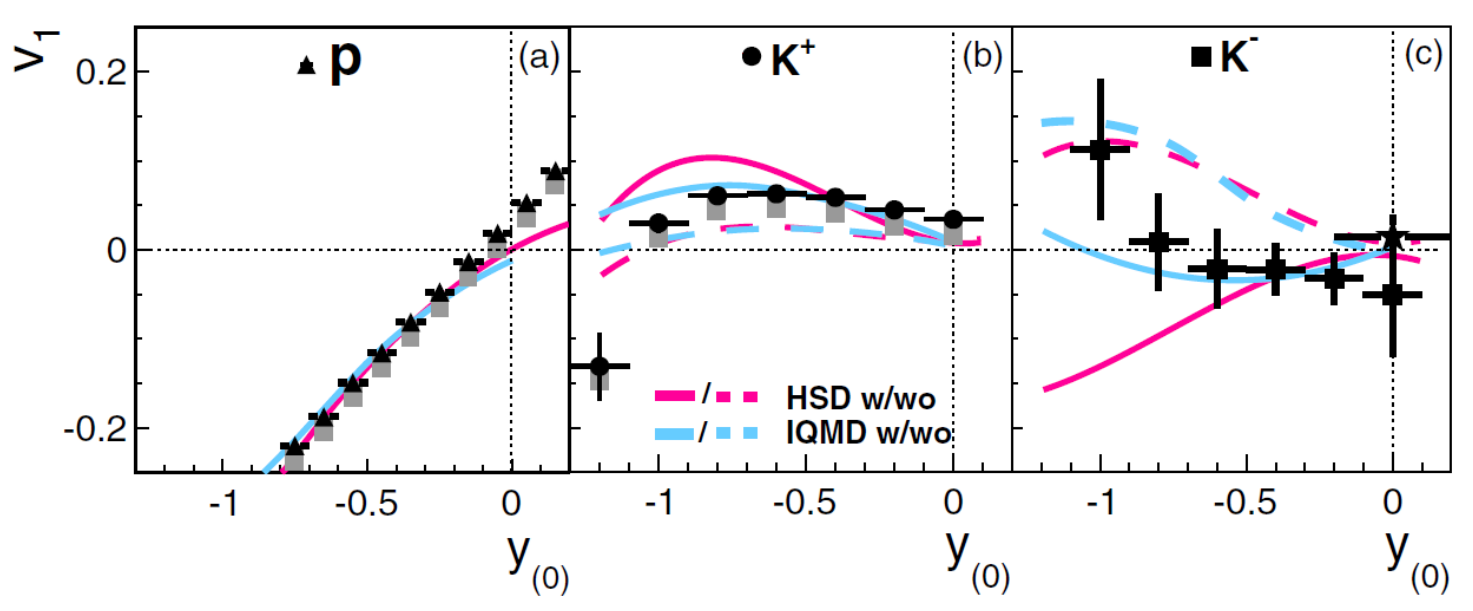

Figure 1: Directed flow $\left(v_{1}\right)$ as function of reduced rapidity for protons, and charged kaons from collisions of $\mathrm{Ni}(1.91 \mathrm{~A} \mathrm{GeV})+\mathrm{Ni}$. Lines depict predictions of the HSD (magenta), and IQMD (blue) transport models with (solid), and without (dashed) the in-medium effects. See text for details. Picture taken from [18].

\section{Experiment}

The S325 experiment was performed by the FOPI Collaboration at the SIS18 accelerator at GSI, Darmstadt. ${ }^{58} \mathrm{Ni}$ ions were accelerated to the beam kinetic energy of $1.91 \mathrm{~A} \mathrm{GeV}$ and collided with the ${ }^{\text {nat }} \mathrm{Ni}$ target.

The target was surrounded by the central drift chamber (CDC) covering the polar angles of $27^{\circ}<\theta_{\text {lab }}<113^{\circ}$. The CDC was encircled by two Time-of-Flight detection systems, the plastic

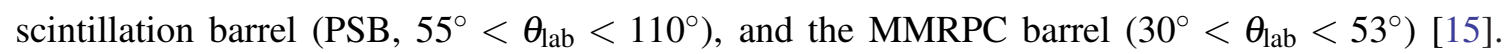
This set of detectors was mounted inside the magnet solenoid delivering the magnetic field of $0.617 \mathrm{~T}$. A wide geometrical acceptance range of the setup, matched with the good (PSB), and excellent (MMRPC) ToF resolution translated into the broad acceptance of phase space available within the back hemisphere with respect to the nucleon-nucleon (NN) centre of mass. The trigger based on the multiplicity of charged particles $[16,17]$ selected events ranging from most central to semi-peripheral collisions $\left(\sigma \approx 56 \% \sigma_{\text {geom }}\right)$. More details on the experimental setup and the identification of kaons can be found in $[18,19]$.

\section{Directed flow of charged kaons}

In this section we summarize the analysis published in [18]. The azimuthal distribution of emitted particles with respect to the reaction plane is often decomposed in terms of the Fourier series,

$$
\frac{d N}{d \phi}=\sum_{i=1}^{\infty} v_{\mathrm{n}} \cos (n \phi)
$$

where $v_{\mathrm{n}}$ are the weights of the subsequent harmonic terms.

The dependence of the $v_{1}$ parameter ("directed flow") of protons and charged kaons on the reduced rapidity in the NN reference frame $\left(y_{(0)}=\left(y_{\mathrm{Lab}}-y_{\mathrm{NN}}\right) / y_{\mathrm{NN}}\right)$, is shown in Fig. 1. While 


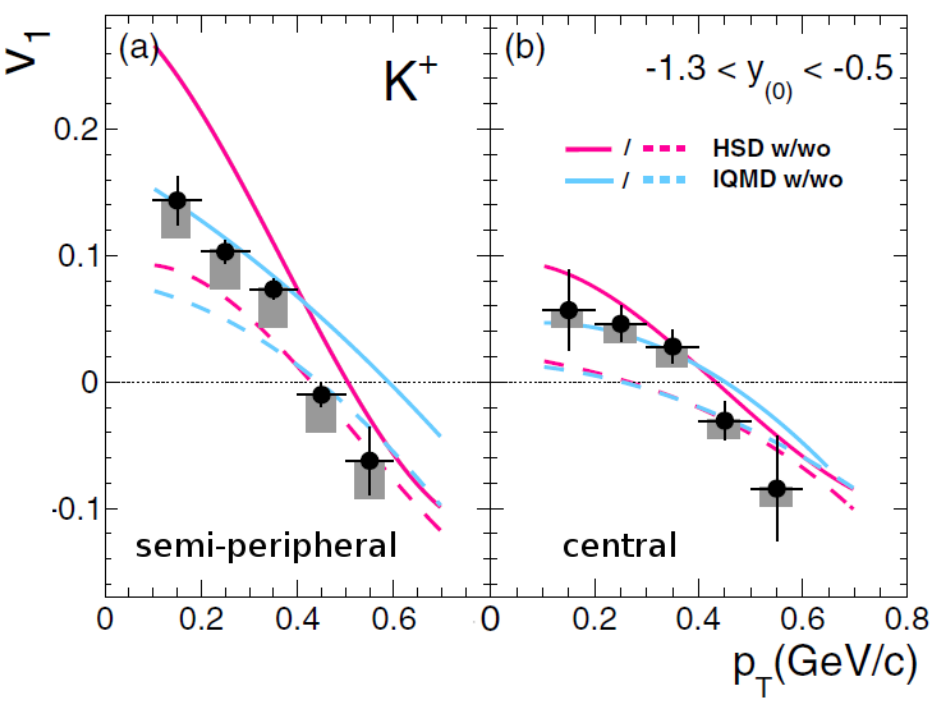

Figure 2: Directed flow $\left(v_{1}\right)$ of $\mathrm{K}^{+}$mesons as function of transverse momentum for (a) peripheral, and (b) central collisions of $\mathrm{Ni}(1.91 \mathrm{~A} \mathrm{GeV})+\mathrm{Ni}$. Lines depict predictions of the HSD (magenta), and IQMD (blue) transport models with (solid), and without (dashed) the in-medium effects. See text for details. Picture taken from [18].

for most rapidities the $\mathrm{K}^{+}$mesons exhibit positive directed flow (for the backward hemisphere), in case of $\mathrm{K}^{-}$mesons the values of $v_{1}$ for the majority of data points are found to be either consistent with 0 or slightly negative. These results were compared to the predictions of two transport models, IQMD [21] and HSD [20], as shown in Fig. 1 with blue, and magenta curves, respectively. The predictions where the in-medium effects were switched off, are depicted by dashed lines. For the IQMD model predictions with the in-medium modifications, the following potentials were chosen: $U_{\mathrm{K}^{+} \mathrm{N}}\left(\rho=\rho_{0}, p=0\right)=+20 \mathrm{MeV}$, and $U_{\mathrm{K}^{-} \mathrm{N}}\left(\rho=\rho_{0}, p=0\right)=-45 \mathrm{MeV}$, where $\rho_{0}$ is the normal nuclear density. It seems, that the calculations assuming the in-medium effects nicely reproduce the $v_{1}\left(y_{(0)}\right)$ pattern, although the choice of slightly smaller values of both $U_{\mathrm{KN}}$ may give a better agreement with the experimental data. Within the HSD model, only the $\mathrm{K}^{+}$in-medium effects are parameterized by the $U_{\mathrm{KN}}$ potential. For the current comparison, it was set to $+20 \mathrm{MeV}$. The $\mathrm{K}^{-}$mesons are treated as off-shell particles within the G-Matrix formalism, and therefore the potential picture should be perceived only as an approximation. For $\mathrm{K}^{-}$the strength of in-medium effects corresponds to $U_{\mathrm{K}^{-} \mathrm{N}}\left(\rho=\rho_{0}, p=0\right)=-50 \mathrm{MeV}$. The model predictions obtained with the abovementioned potentials appear to generate too strong effect with respect to the experimental findings, and indicate that calculations with $U_{\mathrm{KN}}$ about halved could be a better choice.

Fig. 2 shows the dependence of directed flow on transverse momentum for the $\mathrm{K}^{+}$mesons measured within $-1.3<y_{(0)}<-0.5$ range, for semi-peripheral, and central $\mathrm{Ni}+\mathrm{Ni}$ collisions. The comparisons of calculations of the HSD, and IQMD transport models do not yield straightforward conclusions. For the semi-peripheral collisions the IQMD predictions do not seem to favour any of the two scenarios. In case of the in-medium version of the HSD calculations, the predicted $v_{1}$ pattern at low $p_{\mathrm{T}}$ exhibits much stronger flow than that found experimentally, suggesting again, that 


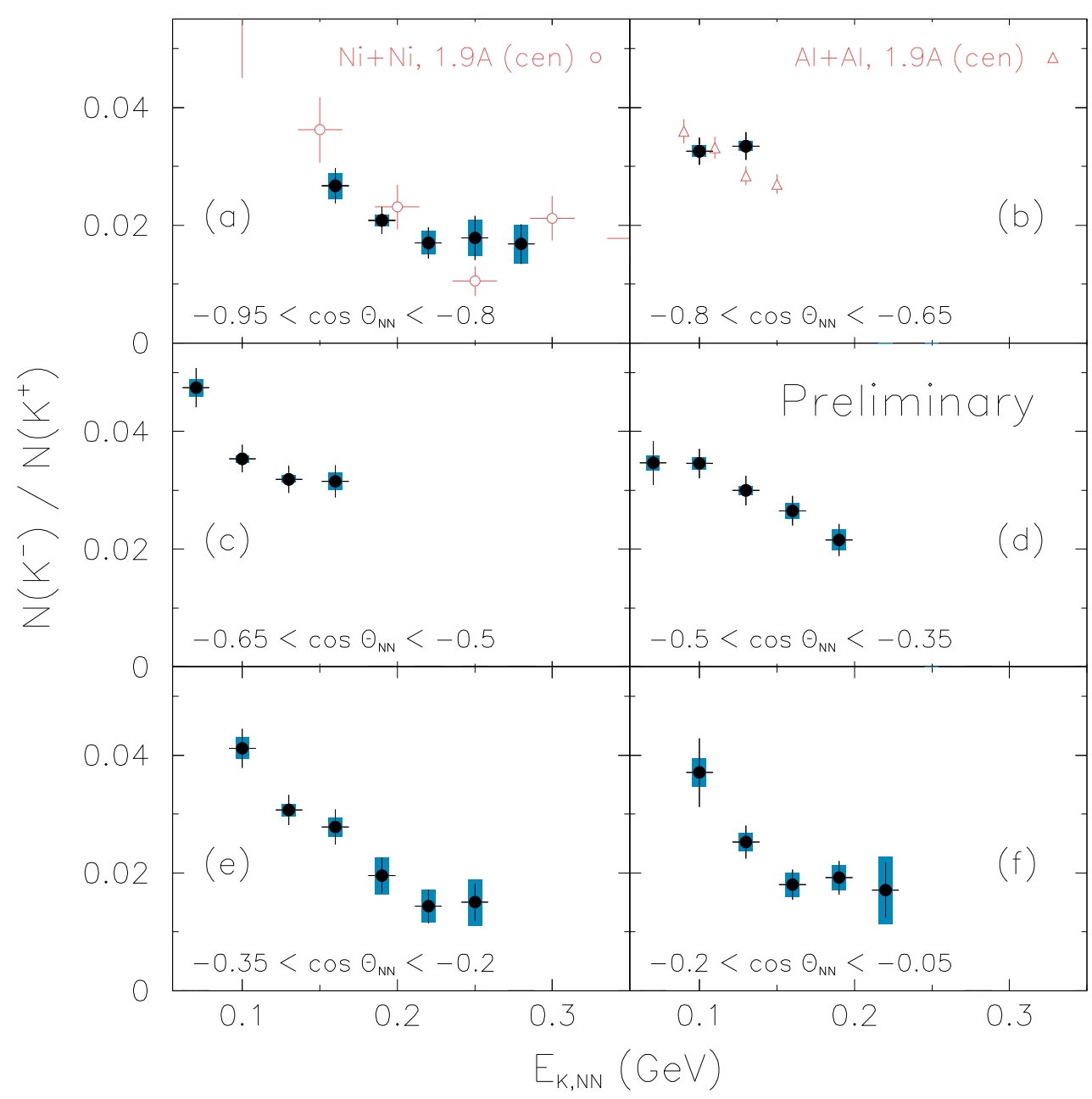

Figure 3: (Full circles:) ratios of $\mathrm{K}^{-} / \mathrm{K}^{+}$as function of kinetic energy for subsequent slices of $\cos \theta_{\mathrm{NN}}$, from s325 experiment. (Open circles:) data for central $\mathrm{Ni}+\mathrm{Ni}$ collisions, from previous experiment, published in [6]. (Open triangles:) data for central Al+Al collisions, published in [8]. See text for details.

the size of potential was too high. For the central collisions, the data points at low $p_{\mathrm{T}}$ turned out to be situated between two scenarios, sugesting again, that about halved value of the tested potential could yield a better agreement. At higher $p_{\mathrm{T}}$ the comparison is inconclusive.

\section{Phase space distribution of $\mathrm{K}^{-} / \mathrm{K}^{+}$ratio}

The reconstructed phase space yield of $\mathrm{K}^{-}$mesons was divided by that of positively charged kaons. In Fig. 3 we show for the first time this ratio as a function of kinetic energy in the NN reference frame for subsequent slices of $\cos \theta_{\mathrm{NN}}$, spanning nearly all the emission angles in the backward hemisphere. The data points for the $\mathrm{K}^{-} / \mathrm{K}^{+}$ratio from the central Ni+Ni collisions at the same beam energy, reported in [6] are shown for rough comparison in panel (a) of this figure. The latter data were measured only within $-0.97<\cos \theta_{\mathrm{NN}}<-0.87$ range, and exhibit clearly 
larger statistical uncertainties than these obtained in the recent experiment. Also, four data points are available for the collisions of $\mathrm{Al}+\mathrm{Al}$ at the same beam energy [8]. As they were measured for the range $-0.87<\cos \theta_{\mathrm{NN}}<-0.72$, they were placed in panel (b) for rough comparison. The systematic errors of our data points have been evaluated at the $1 \sigma$ confidence level, and include variations in the background subtraction procedure, and track quality cuts. Although preliminary, these data are in the final stage of analysis, and the theorists interested to compare this data to the prediction of the transport model are invited to contact us.

\section{Summary}

The collisions of $\mathrm{Ni}+\mathrm{Ni}$ at the beam kinetic energy of $1.91 \mathrm{~A} \mathrm{GeV}$ were measured by the FOPI Collaboration. The excellent time resolution of the MMRPC detector allowed to obtain more precise data on the charged kaon production in terms of the directed flow and the $\mathrm{K}^{-} / \mathrm{K}^{+}$ratio in a wide acceptance. Comparisons of these distributions to the predictions of HSD and IQMD transport models allows to get insight into the scale of modifications of kaon properties in the nucleus-nucleus collision zone.

For $\mathrm{K}^{+}$the $v_{1}\left(y_{(0)}\right)$ study indicates a minor value $U_{\mathrm{K}^{+} \mathrm{N}} \approx 10 \mathrm{MeV}$. Conclusions from the $v_{1}\left(p_{\mathrm{T}}\right)$ study are less clear:

(a) within the central collisions, similarly small in-medium effects are favoured;

(b) for the semi-peripheral collisions, whereas the HSD comparison tends to point to in-medium effect of similar size, within the IQMD calculation none of scenarios seem to follow the experimental trend.

In case of $\mathrm{K}^{-}$, the analysis of $v_{1}\left(y_{(0)}\right)$ dependence seems to indicate some moderate in-medium effect, which in terms of $U_{\mathrm{K}^{-} \mathrm{N}}$ could be approximated by about $-25 \mathrm{MeV}$ (HSD) or about $-45 \mathrm{MeV}$ (IQMD).

\section{References}

[1] D.B. Kaplan and A.E. Nelson, Phys. Lett. B 175, 57 (1986).

[2] G.E. Brown and M. Rho, Phys. Rev. Lett. 66, 2720 (1991).

[3] J. Schaffner-Bielich, I.N. Mishustin, J. Bondorf, Nucl. Phys. A 625, 325 (1997).

[4] W. Weise, Nucl. Phys. A 610, 35c (1996).

[5] M.F.M. Lutz, Prog. Part. Nucl. Phys. 53, 125 (2004).

[6] K. Wiśniewski et al. (FOPI Collaboration), Eur. Phys. J. A 9, 515 (2000).

[7] F. Laue et al. (KaoS Collaboration), Eur. Phys. J. A 9, 397 (2000).

[8] P. Gasik et al. (FOPI Collaboration), Eur. Phys. J. A 52, 177 (2016).

[9] Y. Shin et al. (KaoS Collaboration), Phys. Rev. Lett 81, 1576 (1998).

[10] P. Crochet et al. (FOPI Collaboration), Phys. Lett. B 486, 6 (2000).

[11] G. Agakishiev et al. (HADES Collaboration), Phys. Rev. C 80, 025209 (2009). 
[12] K. Piasecki et al. (FOPI Collaboration), Phys. Rev. C 94, 014901 (2016).

[13] M. Lorenz (HADES Collaboration), PoS (BORMIO2010) 038.

[14] M. Bleicher (UrQMD), results presented in this conference.

[15] M. Kiš et al. (FOPI Collaboration), Nucl. Instr. Meth. A 646, 27 (2011).

[16] A. Gobbi et al. (FOPI Collaboration), Nucl. Instr. Meth. A 324, 156 (1993).

[17] B. Sikora (FOPI Collaboration), Acta Phys. Pol. B 31, 135 (2000).

[18] V. Zinyuk et al. (FOPI Collaboration), Phys. Rev. C 90, 025210 (2014).

[19] K. Piasecki et al. (FOPI Collaboration), Phys. Rev. C 91, 054904 (2015).

[20] W. Cassing, L. Tolós, E.L. Bratkovskaya, A. Ramos, Nucl. Phys. A 727, 59 (2003).

[21] C. Hartnack et al., Phys. Rep. 510, 119 (2012). 